\title{
Analysis on Connotations and Features of Occupational Risks
}

\author{
Wei Huang \\ Beijing University of Chinese Medicine School \\ of Management \\ Beijing, China \\ richer_hw@126.com
}

\author{
Ying Wen \\ Beijing University of Chinese Medicine School \\ of Management \\ Beijing, China \\ wenying18@163.com
}

\author{
Caichun Zhang \\ China Woman's University School of Management \\ Beijing, China \\ joycaichun@139.com
}

\begin{abstract}
Under the social background where risks have become normal, it is of practical significance to study occupational risks for stable social development, sound organization growth and individual self-improvement. This article analyzes connotations and puts forward features of occupational risks by researching and clarifying occupational risks for employees with high risks, which is favorable to holistically understand and recognize occupational risks.
\end{abstract}

\section{Keywords- occupational risks; connotation; features}

\section{INTRODUCTION}

With China joining the WTO and the economic globalization, China is facing the new opportunity of economic construction and social development. At the same time, we became a risky society with risks everywhere, including not only natural and irresistible disasters like earthquake and floods, but also more prominent manmade risks such as economic crimes and terrorism activities which bring about potential miseries to this swift changing society[1]. As the subject of manmade risks humans make sense through their occupations. In another word, occupational risks happen in our work and daily life no matter from the aspect of society, organization or individuals. Therefore, researching on recognition and management of occupational risks is of dramatically practical significance for stable social development, sound organization growth and individual self-improvement.

However, people have had an inadequate understanding and weak precaution consciousness of occupational risks for quite a long time. At present, our researches on occupational risks are insufficient and partial, in which scholars only focus on some specific occupations or positions, such as medical staff, financial staff, legal personnel, senior executives, policepersons, mine workers and other high risky occupations. This article aims at researching and clarifying occupational risks of employees with high risks, and analyzing connotations and features of occupational risks, in order to strengthen people's ability of recognizing occupational risks.

\section{CONNOTATIONS OF OCCUPATIONAL RISKS}

\section{A. The Concept of Occupational Risks}

Occupational risks are initially brought in from abroad to preserve or increase the value of state owned capitals and take the advantage of western modern restraint and incentive mechanisms for managers in market economy [2]. Many domestic scholars have done plentiful researches on occupational risks but only few of them have given the definition and demonstrated its scope. Three major illustrations on occupational risks from different perspectives are as follow, A) from the perspective of society, some kinds of professions are out of need because of the development of social productive forces, social transformation and professional changes. The typical definition refers to the possibility that a labor loses his profession or job under the condition of commodity economy, which is put forward by Li Yuzhu (1988) [3]; B) in terms of industry, as an occupation, it must be under the supervision of the public and once it transgresses it should be punished in some way, and suffers economic or other kinds of losses. So the preconditions and environment for this profession to exist and improve encounter damages. Sun Kun is the first person to formulate the occupational risk of audit as "the aggregation of all actions and environment that have adverse impacts on the existence and development of audit" in 1997 [4]; C) at the aspect of an individual, it means the possibility of facing losses due to doing a specific kind of job. This explanation is widely used, typified by a definition offered by Tao Ye (2012) that it is the risk endured by an employee in a certain frequency during his work [5]. Meanwhile, Chen Shaohui, He Chunsheng and Huang Shuyun (2005) regard the occupational risk in medical industry as the possibility of uncertain damages caused by medical staff when they are fulfilling their responsibilities [6].

It is observed that people shifted from society and industry to individuals in the process of researches. Combining the definition of ISO0 310000 that the risk is "impacts on targets caused by uncertainties", this article also integrates the three above aspects to define occupational risks as the impacts on professional activities and relevant workers, generated by uncertainties during their terms of 
office. This impact is not limited to various losses, but also includes incentives to employees.

\section{B. Types of Occupational Risks}

All researches attempted to define, analyze and illustrate occupational risks are at the basis of the collection of types of occupational risks and losses related to risks. Although there are various kinds of potential factors affecting occupational risks, scholars usually classify occupational risks by sorting out causes, events and effects. These factors can be used in classification system of occupational risks of which causes and effects are mostly selected to clarify.

According to causes of occupational risks, there are ethical risks, behavioral risks and legal risks. Occupational risks are generated from different factors. Those caused by fraudulent conducts, unfulfilled due professional skepticism and other matters related to ethics are ethical risks; those triggering risk accidents and aggravating losses are behavioral risks, resulting from defects of life-long development of a certain profession, fail of standardized operations, and the ignorance and negligence of operators; and those are legal risks due to violation of the law. In addition, ethical risks always accompany with behavioral risks [7].

Based on effects of occupational risks, it can be divided into property risks, personnel risks and liability risks. Personnel risks and liability risks refer to risks to legally bear the liability of compensation attributed to damage property or body of others [8].

Although most scholars emphasize on analyzing occupational risks based on effects, the analysis of causes can facilitate practitioners to understand in what aspects they can take precautions against risks, and decrease the frequency of relevant events or mitigate the damage, so as to achieve the expected goal of total risks. Since the causes of losses are diverse, every factor that may affect must be painstakingly analyzed and recognized. Consequently, it is far from enough to simply divide occupational risks into ethical, behavioral and legal risks. Considering that the occurrence of occupational risks relates to internal and external environment, here classify it as systematic risks and unsystematic risks. Also known as industrial risks or common risks, systematic risks are risks aroused by factors that affect all practitioners, including unique risks of one certain profession and environmental risks. Unsystematic risks or individual risks mean risks that influence the occurrence of unique events of this profession, composed of technical risks and ethical risks [9], while legal risks are generated from external environment of this profession as well as the individual of practitioners.

\section{FEATURES OF OCCUPATIONAL RISKS}

As mentioned above, the occurrence of occupational risks is influenced by both internal and external environment. Similarly, the relevant process of risk management includes environment setting, risk evaluation, risk response, communication and consultation, and surveillance and appraisal. Hence, management of occupational risks also needs to start from setting up environment. Only by timely and effectively recognizing features of occupational risks, can one analyze and evaluate occupational risks, and come up with efficacious response strategy and scientific management. Compared with other risks, occupational risks are featured as follows.

\section{A. Universality and Particularity}

Universality means that occupational risks may happen during every professional process. Thus, it is pregnant to seek the common features of occupational risks to strengthen people's ability of precaution. On the other hand, since different professions present various risks with diverse forms, occupational risks show particularity.

\section{B. Inevitability and Contingency}

Inevitability equals to an objective existence determined by features of certain professions, which is also aroused by the diversity of external environment and practitioners, and moral trait and subjective factors of practitioners. However, the inevitability of occupational risks is represented by contingency. In other words, the specific time, places and forms when occupational risks happen are accidental.

\section{Potentiality and Reality}

Potentiality refers to the possibility and uncertainty of risks determined by inevitability and contingency of occupational risks. However, results of risks are potential, which may occur during the whole process of professional growth and development. As long as this kind of possibility appears to be objective reality through specific time, places and forms, occupational risks become veritable professional fault or crime.

\section{Controllability and Uncontrollability}

With respect to causes of occupational risks, not only objective factors like unique risks and environmental risks can generate occupational risks, but also subjective factors like practitioners' experience, capability, sense of responsibility and competence can. In terms of occupational risks, objective factors are of difficulty to control because they are independent of man's will. On the other hand, as regards subjective factors, people can enhance professional self-disciplines and accomplishment, and avoid, lessen or transfer risks by utilizing his own experience to recognize risks and taking relevant measures.

It is obvious that occupational risks are objective with huge potential damages, so we need to prevent, avoid and address when they happen. To promote controllability, we need to strengthen awareness of precaution and frequently guard against risks, especially take special measures in cope with the field, industry and personnel with high risk. In order to facilitate individual comprehensive quality and his capability of fighting against risks, society needs to establish and improve warning, prevention and response mechanisms, and boost pre-warning publicity and education to cultivate excellent work style. We also need to confirm professional obligation and strictly formulate occupational disciplines to promote occupational standardized management and reasonably mitigate risks. Personnel should enhance professional self-confidence to actively decrease occupational risks. 


\section{REFERENCES}

[1] Deng Guoliang. AnInterpretation of police professional risksand its prevention[J].Journalof jiangxxi police institute,2011,01:18-24.

[2] Luo Jingfen. Ways of Corporate Reformation, on Establishment of Occupational Risks Mechanism [J]. Review of Economic Research,1994,20:33-37.

[3] Li Yuzhu. Establish and Perfect Occupational Risks Mechanism for Socialist Society [J]. Fujian Tribune (Economics and Sociology),1988,02:52-54.

[4] Sun Kun. On Occupational Risks of Audit [J]. Auditing Research,1997,04:41-45.

[5] Tao Ye. Research Process on Awareness of Occupational Risks for Nursing [J]. Nursing Practice and Research,2012,v.906:116-118.

[6] Chen Shaohui, He Chunsheng, Huang Shuyun. Mechanisms for Guarding Against and Dissolving Risks of the Medical Profession [J]. Chinese Journal of Hospital Administration,2005,08:521-526.

[7] Zhang Linlin. On the Abnormal Occupational Hazards for Chinese Defense Lawyers, Journal of Shandong Public Security College, 2005.5 (3) : 49.

[8] Luo Xiaoyuan, Liu Xiaolian, Yin Dequan. Discussions on Establishing Occupational Risk Fund for CPA [J]. Chinese Institute of Certified Public Accountants, 1998,06:31-40.

[9] Zhuang Kexin, Chen Wen. Meager Opinion on Occupational Risks and its Evaluation of Accountant [J]. China Township Enterprises Accounting,2008,06:185-186. 